ISSN: 2599-3496 print

ISSN: 2614-2376 online

\title{
Understanding the Codex Standard to Ensure Safety and Quality of Palm Oil
}

\author{
Purwiyatno Hariyadi* \\ IPB University, Bogor 16680, Indonesia
}

\begin{abstract}
Indonesia is the number one producer and exporter of palm oil globally. About $85 \%$ of palm oil is used for food applications. Consequently, issues related to food safety and nutrition have always been a major concern, both for producers and consumers of palm oil. In this respect, the palm oil industry must put serious effort to comply with food safety and quality standards, such as those of Codex standards, especially specified at Codex Standard for Named vegetable Oils (CXS 210-1999).
\end{abstract}

Key words: International trade.

\section{INTRODUCTION}

Indonesia is situated nicely at the equator so that the climate is suitable for the optimum growth of oil palm trees, tropical crops producing edible oil. Potentially, productivity of palm oil tree may reach about 8 tons oil/hectare /year in most regions. However, current average actual yields are about 3.3 tons/hectare /year.

This productivity is much higher as compared to that of soybean oil (only 0.4 tons/hectare) and sunflower seed oil (0.5 tones/hectare). Not only that the productivity is high, but palm oil also has a longer productive life span ( $\geq 25$ years). Palm oil also have a cheaper production costs, of about US\$160/ton, compared to that of soybean of about US\$300/ton (Wisena et al. 2014).

According to the Indonesian Palm Oil Association (GAPKI 2020), Indonesia's palm oil production in 2019 was 51.8 million tons, comprising of crude palm oil (CPO) for about 47.2 million tons, and palm kernel oils (PKO) for about 4.6 million tons. This number, as presented at Table 1, is an increased by about 9 percent as compared to the 2018 production of 47.4 million tons.

Currently, Indonesia is not only the biggest producer, but also the main exporter of palm oil and palm derivative products, amounted up to 23.175 million tons of

*Corresponding author:

Department of Food Science \& Technology, Faculty of Agricultural Engineering \& Technology, IPB University, Bogor 16680, Indonesia.

Southeast Asian Food and Agricultural Science and Technology Center, IPB University, Bogor 16680, Indonesia. E-mail: phariyadi@apps.ipb.ac.id. 
Table 1 Indonesian palm oil production and utilization (GAPKI 2020)

\begin{tabular}{lcccccccc}
\hline Year & \multicolumn{2}{c}{$\begin{array}{c}\text { Production } \\
\text { (million tons) }\end{array}$} & \multirow{2}{*}{$\begin{array}{c}\text { Domestic } \\
\text { market }\end{array}$} & \multicolumn{3}{c}{$\begin{array}{c}\text { Export } \\
\text { (million tons) }\end{array}$} & Stock \\
\cline { 2 - 7 } & Total & CPO & PKO & & Total & CPO & $\begin{array}{c}\text { Derivative } \\
\text { Products }\end{array}$ & \\
\hline 2018 & 47.388 & 43.108 & 4.280 & 13.491 & 34.707 & 6.561 & 28.145 & 3.261 \\
\hline 2019 & 51.828 & 47.180 & 4.648 & 16.673 & 36.175 & 7.065 & 29.110 & 4.597 \\
\hline
\end{tabular}

${ }^{*}$ Include oleochemicals and biodiesel

palm oil product in 2019. Driven by an increase in global population and economic growth, the demand for vegetable oil over the next decade will continue to increase. According to Oil Market Research (2020), the palm oil sector will continue growing, and anticipated to reach a total of 98.82 million tons in 2024, with growing rate at about $5.9 \%$ (for the period of 2019 to 2024).

\section{THE IMPORTANCE OF CODEX STANDARDS FOR PALM OILS}

Globally, most of the palm oil produced (around $85 \%$ ) is currently used for food applications, especially as a cooking oil and other food ingredients for most margarine, ice cream and many varieties of ready-to-eat meals. This paper suggests, first thing first, that palm oil sector need to ensure the safety aspect of their product.

Food and Agricultural Organization (FAO 2019) has advocated that "if it isn't safe; it isn't foods." This suggests that palm oil used for food application must comply with the food safety standard. For Indonesia, since palm oil is internationally traded, then complying with international food safety standard, i.e., Codex Standards which are developed by the Codex Alimentarius Commission or CAC, is paramount important. The following section of the paper will discuss several selected
Codex standards relevant to ensure safety and quality of palm oil.

\section{CODEX STANDARD FOR NAMED VEGETABLE OILS, CXS 210-1999}

This standard (CXS 210-1999) cover many kinds of named vegetables oil. Especially for palm oil, this standard also provide specific provision for several type of palm oil commonly traded, such as palm oil, palm olein, palm stearin, palm kernel oil, and including the specialty kind such as super olein and palm oil with a higher content of oleic acid (Table 2). The provision described at this Standard applies to the palm oils presented in a state for human consumption, meaning that this standard specifically applicable for palm oil for food applications.

The standard also provides description of essential composition of fatty acid for each type of palm oil product (expressed as percentages) as determined by gas liquid chromatography, as described at Table 3. It was mentioned that palm oil product having fatty acid composition within the appropriate ranges specified in Table 3 follow this Codex standard. It is noted as well that in trade practice, it is customary that a supplementary criterion, for example national geographical and/or climatic variations, may be considered, as necessary, to confirm that a sample 
Table 2 Several type of palm oil covered at the Codex Standard for Named Vegetable Oils (CXS 210-1999)

\begin{tabular}{|c|c|c|}
\hline $\begin{array}{l}\text { Type of palm Oil } \\
\text { Products }\end{array}$ & Description of product & Essential quality factors \\
\hline Palm oil & $\begin{array}{l}\text { Oil derived from the fleshy mesocarp of } \\
\text { the fruit of the oil palm (Elaeis guineen- } \\
\text { sis) }\end{array}$ & \\
\hline Palm olein & $\begin{array}{l}\text { The liquid fraction derived from the frac- } \\
\text { tionation of palm oil }\end{array}$ & $\begin{array}{l}\text { Slip melting point of not } \\
\text { more than } 24^{\circ} \mathrm{C}\end{array}$ \\
\hline Palm stearin & $\begin{array}{l}\text { The high-melting fraction derived from } \\
\text { the fractionation of palm oil }\end{array}$ & $\begin{array}{l}\text { Slip melting point of not } \\
\text { less than } 44^{\circ} \mathrm{C}\end{array}$ \\
\hline Palm super olein & $\begin{array}{l}\text { The liquid fraction derived from palm oil } \\
\text { (described above) produced through a } \\
\text { specially controlled crystallization pro- } \\
\text { cess to achieve an iodine value of } 60 \text { or } \\
\text { higher }\end{array}$ & $\begin{array}{l}\text { Slip melting point of not } \\
\text { more than } 19.5^{\circ} \mathrm{C}\end{array}$ \\
\hline Palm kernel oil & $\begin{array}{l}\text { Oil derived from the kernel of the fruit of } \\
\text { the oil palm (E. guineensis). }\end{array}$ & \\
\hline Palm kernel olein & $\begin{array}{l}\text { The liquid fraction derived from fraction- } \\
\text { ation of palm kernel oil }\end{array}$ & $\begin{array}{l}\text { Slip melting point between } \\
21 \text { to } 26^{\circ} \mathrm{C}\end{array}$ \\
\hline Palm kernel stearin & $\begin{array}{l}\text { The solid fraction derived from frac- } \\
\text { tionation of palm kernel oil (described } \\
\text { above). }\end{array}$ & $\begin{array}{l}\text { Slip melting point between } \\
31 \text { to } 34^{\circ} \mathrm{C}\end{array}$ \\
\hline $\begin{array}{l}\text { Palm oil with a high- } \\
\text { er content of oleic } \\
\text { acid }\end{array}$ & $\begin{array}{l}\text { Oil derived from the fleshy mesocarp of } \\
\text { hybrid palm fruit }(\mathrm{O} \times \mathrm{G})(E \text {. oleifera } \times E \text {. } \\
\text { guineensis) }\end{array}$ & $\begin{array}{l}\text { Palm oil with a higher } \\
\text { content of oleic acid must } \\
\text { contain not less than } 48 \% \\
\text { oleic acid (as \% of total } \\
\text { fatty acids) }\end{array}$ \\
\hline
\end{tabular}

is following the Codex Standard. In addition to several essential composition and quality factors for each type of palm oil described at Table 2 and Table 3, the standard also provides detail provision, including food additives, contaminants, hygiene, labelling and methods of analysis and sampling.

This standard also comes with appendix containing other quality and composition factors of palm oil product, added as a supplementary information necessary to the essential provision in the standard. A palm oil product, which meets the es- sential provision but does not meet this supplementary information, may still be considered to conform to the standard. These supplementary quality factor and composition of palm oil products can be seen at Table 4, 5 and 6.

Beside information regarding the physical and chemical composition, the appendix of the standard also stated that the colour, odour, and taste of each product shall indicate the.characteristic of the designated product. It shall be free from foreign and rancid odour and taste. Specifically, the maximum level of Fe at crude 
palm kernel olein and crude palm kernel stearin also specified as 5.0 and $7.0 \mathrm{mg} /$ $\mathrm{kg}$, respectively. Provision of free fatty acid also given for virgin palm oil $(5.0 \%$ as palmitic acid) and crude palm kernel oil (4.0\% as lauric acid). Specific requirement for the total carotenoids (as beta-carotene) was also stated, especially for unbleached palm oil, unbleached palm olein and unbleached palm stearin, and should be in the range of 500-2000, 5502500 and $300-1500 \mathrm{mg} / \mathrm{kg}$, respectively.

\section{OTHER RELATED CODEX STANDARDS}

Other than Codex Standard for named vegetable oils (CXS 210-1999) reviewed above, palm oil industry shall also comply with two (2) code of practices, namely (i) code of practice for the storage and transport of edible fats and oils in bulk (CXC $36-1987$ ) and (ii) code of practice for the reduction of 3-monochloropropane-1,2diol esters (3-MCPDE) and glycidyl es-

Table 3 Essential composition of fatty acid of palm oil products as determined by gas liquid chromatography from authentic samples (expressed as percentage of total fatty acids)

\begin{tabular}{|c|c|c|c|c|c|c|c|c|}
\hline $\begin{array}{l}\text { Fatty } \\
\text { acid }\end{array}$ & Palm oil & $\begin{array}{c}\text { Palm } \\
\text { stearin }^{2}\end{array}$ & $\begin{array}{l}\text { Palm } \\
\text { olein }^{2}\end{array}$ & $\begin{array}{l}\text { Palm su- } \\
\text { perolein }\end{array}$ & $\begin{array}{c}\text { Palm } \\
\text { kernel oil }\end{array}$ & $\begin{array}{l}\text { Palm } \\
\text { kernel } \\
\text { stearin }^{3}\end{array}$ & $\begin{array}{l}\text { Palm } \\
\text { kernel } \\
\text { olein }^{3}\end{array}$ & $\begin{array}{c}\text { Palm oil } \\
\text { with a } \\
\text { higher } \\
\text { oleic acid }\end{array}$ \\
\hline C6:0 & ND & ND & ND & ND & ND-0.8 & ND-0.2 & ND-0.7 & ND \\
\hline C8:0 & ND & ND & ND & ND & $2.4-6.2$ & $1.3-3.0$ & $2.9-6.3$ & ND \\
\hline C10:0 & ND & ND & ND & ND & $2.6-5.0$ & 2.4-3.3 & $2.7-4.5$ & ND \\
\hline C12:0 & ND-0.5 & $0.1-0.5$ & $0.1-0.5$ & $0.1-0.5$ & $45.0-55.0$ & $52.0-59.7$ & $39.7-47.0$ & ND-0.5 \\
\hline $\mathrm{C} 14: 0$ & $0.5-2.0$ & $1.0-2.0$ & $0.5-1.5$ & $0.5-1.5$ & $14.0-18.0$ & $20.0-25.0$ & $11.5-15.5$ & ND-0.8 \\
\hline C16:0 & 39.3-47.5 & $48.0-74.0$ & $38.0-43.5$ & $30.0-39.0$ & $6.5-10.0$ & $6.7-10.0$ & 6.1-10.6 & $23.0-38.0$ \\
\hline C16:1 & ND-0.6 & ND-0.2 & ND-0.6 & ND-0.5 & ND-0.2 & ND & ND-0.1 & ND-0.8 \\
\hline C17:0 & ND-0,2 & ND-0.2 & ND-0.2 & ND-0.1 & ND & ND & ND & ND-0.2 \\
\hline C17:1 & ND & ND-0.1 & ND-0.1 & ND & ND & ND & ND & ND \\
\hline C18:0 & $3,5-6.0$ & $3.9-6.0$ & $3.5-5.0$ & $2.8-4.5$ & $1.0-3.0$ & $1.0-3.0$ & $1.7-3.0$ & $1.5-4.5$ \\
\hline C18:1 & $36.0-44.0$ & $15.5-36.0$ & $39.8-46.0$ & $43.0-49.5$ & $12.0-19.0$ & $4.1-8.0$ & 14.4-24.6 & $48.0-60.0$ \\
\hline C18:2 & $9.0-12.0$ & $3.0-10.0$ & $10.0-13.5$ & $10.5-15.0$ & $1.0-3.5$ & $0.5-1.5$ & $2.4-4.3$ & $9.0-17.0$ \\
\hline C18:3 & ND-0.5 & ND-0.5 & ND-0.6 & $0.2-1.0$ & ND-0.2 & ND-0.1 & ND-0.3 & ND-0.6 \\
\hline C20:0 & ND-1.0 & ND-1.0 & ND-0.6 & ND-0.4 & ND-0.2 & ND-0.5 & ND-0.5 & ND-0.4 \\
\hline C20:1 & ND-0.4 & ND-0.4 & ND-0.4 & ND-0.2 & ND-0.2 & ND-0.1 & ND-0.2 & ND-0.2 \\
\hline C20:2 & ND & ND & ND & ND & ND & ND & ND & ND-0.5 \\
\hline $\mathrm{C} 22: 0$ & ND-0.2 & ND-0.2 & ND-0.2 & ND-0.2 & ND-0.2 & ND & ND & ND-0.3 \\
\hline $\mathrm{C} 22: 1$ & ND & ND & ND & ND & ND & ND & ND & ND \\
\hline $\mathrm{C} 22: 2$ & ND & ND & ND & ND & ND & ND & ND & ND \\
\hline C24:0 & ND & ND & ND & ND & ND & ND & ND & ND-0.2 \\
\hline $24: 1$ & ND & ND & ND & ND & ND & ND & ND & ND \\
\hline
\end{tabular}

ND - non detectable, defined as $<0.05 \%$; ${ }^{2}$ Fractionated product from palm oil; ${ }^{3}$ Fractionated product from kernel oil. 
Table 4 Chemical and physical characteristics of crude oil (see Appendix of the Codex Standard for Named Vegetable Oils (CXS 210-1999))

\begin{tabular}{|c|c|c|c|c|c|c|c|c|}
\hline $\begin{array}{l}\text { Chemical and } \\
\text { physical char- } \\
\text { acteristics of } \\
\text { crude palm oil }\end{array}$ & Palm Oil & $\begin{array}{l}\text { Palm } \\
\text { Olein }\end{array}$ & $\begin{array}{c}\text { Palm } \\
\text { Stearin }\end{array}$ & $\begin{array}{l}\text { Palm } \\
\text { Supero- } \\
\text { lein }\end{array}$ & $\begin{array}{c}\text { Palm } \\
\text { Kernel } \\
\text { Oil }\end{array}$ & $\begin{array}{l}\text { Palm } \\
\text { Kernel } \\
\text { Olein }\end{array}$ & $\begin{array}{l}\text { Palm } \\
\text { Kernel } \\
\text { Steain }\end{array}$ & $\begin{array}{c}\text { Palm Oil } \\
\text { with high- } \\
\text { er oleic } \\
\text { acid } \\
\end{array}$ \\
\hline $\begin{array}{l}\text { Relative } \\
\text { density }\left(\mathrm{X}^{\circ} \mathrm{C} /\right. \\
\text { water at } \\
\left.20^{\circ} \mathrm{C}\right)\end{array}$ & $\begin{array}{l}0.891- \\
0.899\end{array}$ & $\begin{array}{l}0.899- \\
0.920\end{array}$ & $\begin{array}{l}0.881- \\
0.891\end{array}$ & $\begin{array}{l}0.900- \\
0.925\end{array}$ & $\begin{array}{l}0.899- \\
0.914\end{array}$ & $\begin{array}{l}0.906- \\
0.909\end{array}$ & $\begin{array}{l}0.902- \\
0.908\end{array}$ & $\begin{array}{l}0.896- \\
0.910\end{array}$ \\
\hline $\begin{array}{l}\text { Apparent } \\
\text { density }(\mathrm{g} / \mathrm{ml})\end{array}$ & $\begin{array}{l}0.889- \\
0.895 \\
\text { at } 50^{\circ} \mathrm{C} \\
\end{array}$ & $\begin{array}{l}0.896- \\
898 \\
\text { at } 40^{\circ} \mathrm{C}\end{array}$ & $\begin{array}{l}0.881- \\
0.885 \\
\text { at } 60^{\circ} \mathrm{C}\end{array}$ & $\begin{array}{l}0.886- \\
0.900 \\
\text { at } 40^{\circ} \mathrm{C} \\
\end{array}$ & - & $\begin{array}{l}0.904- \\
0.907\end{array}$ & $\begin{array}{l}0.904- \\
0.906\end{array}$ & ND \\
\hline $\begin{array}{l}\text { Refractive } \\
\text { Index } \\
\left(\mathrm{ND} 40^{\circ} \mathrm{C}\right)\end{array}$ & $\begin{array}{l}1.454- \\
1.456 \\
\text { at } 50^{\circ} \mathrm{C} \\
\end{array}$ & $\begin{array}{l}1.458- \\
1.460\end{array}$ & $\begin{array}{l}1.447- \\
1.452 \\
\text { at } 60^{\circ} \mathrm{C}\end{array}$ & $\begin{array}{l}1.459- \\
1.460\end{array}$ & $\begin{array}{l}1.448- \\
1.452\end{array}$ & $\begin{array}{l}1.541- \\
1.453\end{array}$ & $\begin{array}{l}1.449- \\
1.451\end{array}$ & $\begin{array}{l}1.459- \\
1.462\end{array}$ \\
\hline $\begin{array}{l}\text { Saponification } \\
\text { value (mg } \\
\mathrm{KOH} / \mathrm{g} \text { oil) }\end{array}$ & $190-209$ & $\begin{array}{l}194- \\
202\end{array}$ & $\begin{array}{l}193- \\
305\end{array}$ & $\begin{array}{l}180- \\
205\end{array}$ & $230-254$ & $231-244$ & $244-255$ & 189-199 \\
\hline lodine value & $50.0-55.0$ & 56 & $<48$ & $\geq 60$ & $\begin{array}{l}14.1- \\
21.0\end{array}$ & $20-28$ & $4-8.5$ & $58-75$ \\
\hline $\begin{array}{l}\text { Unsaponifi- } \\
\text { able matter } \\
(\mathrm{g} / \mathrm{kg})\end{array}$ & $\leq 12$ & $\leq 13$ & $\leq 9$ & $\leq 13$ & $\leq 10$ & $\leq 15$ & $\leq 15$ & $\leq 12$ \\
\hline
\end{tabular}

ters (GE) in refined oils and food products made with refined oils (CXC 79-2019).

In addition, to ensure the general safety and quality provision of palm oil as food, several other general Codex guidelines, code of practices and/or standards shall also be consulted and implemented. Specifically, palm oil stakeholders should pay closer attention to the latest revised version of General Principles of Food Hygiene (CXC 1-1969) including Good Hygiene Practices (GHPs) and the Hazard Analysis and Critical Control Point (HACCP) System, General Principles for the Addition of Essential Nutrients to Foods (CXG 9-1987), General Standard for Contaminants and Toxins in Food and Feed -(CXS 193-1995), and also General Standard for Food Additives (CXS
192-1995). Complying to those general Codex guidelines, code of practices and/ or standards will not only provide better assurance of safety but also facilitate international trade of palm oil.

\section{CONCLUSIONS}

The opportunities and potentials for the palm oil sector to play a strategic role in Indonesia's development are enormous. However, this potential can only be realized by appropriately complying the Codex standards, especially in ensuring safety and quality of palm oil. This is not only importance for Indonesia as the biggest palm oil exporting country, but also as the palm oil consuming country. All palm oil stakeholders need 
Table 5 Levels of desmethylsterols in crude vegetable oils from authentic samples as a percentage of total sterols

\begin{tabular}{|c|c|c|c|c|c|c|c|c|}
\hline $\begin{array}{l}\text { Level/ com- } \\
\text { position }\end{array}$ & Pam oil & $\begin{array}{l}\text { Palm } \\
\text { olein }\end{array}$ & $\begin{array}{l}\text { Palm } \\
\text { stearin }\end{array}$ & $\begin{array}{l}\text { Palm su- } \\
\text { perolein }\end{array}$ & $\begin{array}{l}\text { Palm } \\
\text { kernel } \\
\text { oil }\end{array}$ & $\begin{array}{l}\text { Palm } \\
\text { kernel } \\
\text { olein }\end{array}$ & $\begin{array}{c}\text { Palm } \\
\text { kernel } \\
\text { stearin }\end{array}$ & $\begin{array}{c}\text { Palm oil } \\
\text { with high- } \\
\text { er olein } \\
\text { acid }\end{array}$ \\
\hline Cholesterol & $2.6-6.7$ & $2.6-7.0$ & $2.5-5.0$ & $2.0-3.5$ & $0.6-3.7$ & $1.5-1.9$ & $1.4-1.7$ & $1.7-4.7$ \\
\hline $\begin{array}{l}\text { Brassicast- } \\
\text { erol }\end{array}$ & ND & ND & ND & ND & ND-0.8 & ND-0.2 & ND-2.2 & ND-0.4 \\
\hline $\begin{array}{l}\text { Campes- } \\
\text { terol }\end{array}$ & $18.7-27.5$ & $12.5-39.0$ & $15.0-26.0$ & $22.0-26.0$ & $8.4-12.7$ & $7.9-91$ & $8.2-9.7$ & $16.6-21.9$ \\
\hline $\begin{array}{l}\text { Stigmas- } \\
\text { terol }\end{array}$ & $8.5-13.9$ & $7.0-18.9$ & $9.0-15.0$ & $18.2-20.0$ & $\begin{array}{l}12.0- \\
16.9\end{array}$ & $\begin{array}{l}13.4- \\
14.7\end{array}$ & $\begin{array}{l}14.1- \\
15.0\end{array}$ & $11.2-15.5$ \\
\hline $\begin{array}{l}\text { Beta-sitos- } \\
\text { terol }\end{array}$ & $50.2-62.1$ & $45.0-71.0$ & $50.0-60.0$ & $55.0-70.0$ & $\begin{array}{l}62.6- \\
73.1\end{array}$ & $\begin{array}{l}67.1- \\
69.2 \\
\end{array}$ & $\begin{array}{l}67.0- \\
70.0\end{array}$ & $57.2-67.0$ \\
\hline $\begin{array}{l}\text { Delta-5-av- } \\
\text { enasterol }\end{array}$ & ND-2.8 & ND-3.0 & ND-3.0 & $0-1.0$ & $1.4-9.0$ & $3.3-4.6$ & $33-4.1$ & ND-1.9 \\
\hline $\begin{array}{l}\text { Delta-7-stig- } \\
\text { mastenol }\end{array}$ & $0.2-2.4$ & ND-3.0 & ND-3.0 & 0.0 .3 & ND-2.1 & ND-0.6 & ND-0.3 & ND-0.2 \\
\hline $\begin{array}{l}\text { Delta-7-av- } \\
\text { enasterol }\end{array}$ & ND-5.1 & ND-6.0 & ND-3.0 & $0-0.3$ & ND-1.4 & ND-0.5 & ND-0.3 & ND-1.0 \\
\hline Others & ND & ND-10.4 & ND-5.0 & $0-2.0$ & ND-2.7 & $2.9-3.7$ & $1.0-3.0$ & ND-3.8 \\
\hline $\begin{array}{l}\text { Total sterol } \\
(\mathrm{mg} / \mathrm{kg})\end{array}$ & $300-700$ & $270-800$ & $250-500$ & 100 & $\begin{array}{l}700- \\
1400 \\
\end{array}$ & $\begin{array}{l}816- \\
1339 \\
\end{array}$ & $\begin{array}{l}775- \\
1086 \\
\end{array}$ & $519-1723$ \\
\hline
\end{tabular}

to work together to establishing safety and quality assurance system, covering the entire supply chain (production, handling, transportation, and service chains) to ensure safety and quality of palm oil produced, consumed, and traded.

\section{REFERENCES}

Code of Practice (COP) for the reduction of 3-Monochloropropane-1,2- diol esters (3-MCPDE) and Glycidyl Esters (GE) in refined oils and food products made with refined oils (CXC 79-2019). Available at http://www.fao.org/fao-who-codexalimentarius/codex-texts/codes-ofpractice/en/.
Code of Practice for the Storage and Transport of Edible Fats and Oils in Bulk (CXC 36 - 1987). Available at http://www.fao.org/fao-who-codexalimentarius/codex-texts/codes-of-practice/en/.

Codex Standard for Named vegetable Oils (CXS 210-1999) Available at http:// www.fao.org/fao-who-codexalimentarius/codex-texts/list-standards/en/.

FAO. 2019. If it isn't safe, it isn't food: Six ways we can work together to prevent foodborne diseases. Available at http://www.fao.org/fao-stories/ article/en/c/1179647/\#: :text=A\%20 healthy $\% 20$ diet $\% 20$ starts $\% 20$ with,can $\% 20$ even $\% 2$ lead $\% 20$ to $\% 20$ death. \&text $=F A O \% 20$ is $\% 20$ the $\% 20$ 
Table 6 Levels of tocopherols and tocotrienols in crude vegetable oils from authentic samples $(\mathrm{mg} / \mathrm{kg})$

\begin{tabular}{llccccccc}
\hline $\begin{array}{l}\text { Level/ } \\
\begin{array}{l}\text { composi- } \\
\text { tion }\end{array}\end{array}$ & Pam Oil & $\begin{array}{c}\text { Palm } \\
\text { Olein }\end{array}$ & $\begin{array}{c}\text { Palm } \\
\text { Stearin }\end{array}$ & $\begin{array}{c}\text { Palm Su- } \\
\text { perolein }\end{array}$ & $\begin{array}{c}\text { Palm } \\
\text { Kernel } \\
\text { Oil }\end{array}$ & $\begin{array}{c}\text { Palm } \\
\text { Kernel } \\
\text { Olein }\end{array}$ & $\begin{array}{c}\text { Palm } \\
\text { Kernel } \\
\text { Stearin }\end{array}$ & $\begin{array}{c}\text { Palm Oil } \\
\text { with high- } \\
\text { er olein } \\
\text { acid }\end{array}$ \\
\hline $\begin{array}{l}\text { Alpha- To- } \\
\text { copherol }\end{array}$ & $4-193$ & $30-280$ & ND-100 & $130-240$ & ND-44 & ND-11 & ND-10 & $49-188$ \\
\hline $\begin{array}{l}\text { Betta-To- } \\
\text { copherol }\end{array}$ & ND-234 & ND-250 & ND-50 & ND-40 & ND-248 & ND-6 & ND-2 & ND \\
\hline $\begin{array}{l}\text { Gamma- } \\
\text { Tocopherol }\end{array}$ & ND-526 & ND-100 & ND-50 & ND-40 & ND-257 & ND-3 & ND-1 & $4-138$ \\
\hline $\begin{array}{l}\text { Delta- To- } \\
\text { copherol }\end{array}$ & ND-123 & ND-100 & Nd-50 & ND-30 & ND & ND-4 & ND & ND-31 \\
\hline $\begin{array}{l}\text { Alpha-to- } \\
\text { cotrienol }\end{array}$ & $4-336$ & $50-500$ & $20-150$ & $170-300$ & ND & ND-70 & ND-73 & $74=256$ \\
\hline $\begin{array}{l}\text { Gamma- } \\
\text { Tocotrienol }\end{array}$ & $14-710$ & $20-700$ & $10-500$ & $230-420$ & ND-60 & $1-10$ & ND-8 & $406-887$ \\
\hline $\begin{array}{l}\text { Delta-to- } \\
\text { cotrienol }\end{array}$ & ND-377 & $40-120$ & $5-150$ & $60-120$ & ND & ND-2 & ND-1 & $33-86$ \\
\hline $\begin{array}{l}\text { Total } \\
\text { (mg/kg) }\end{array}$ & $150-1500$ & $300-1800$ & $100-700$ & $400-1400$ & ND-260 & ND-90 & ND-89 & $562-1471$ \\
\hline
\end{tabular}

only $\% 20$ international, aspects $\% 20$ of\%20the\%20food\%20chain.

GAPKI. 2020. Reflection of palm oil industry 2019 and prospects of 2020. (In Bahasa Indonesia: Refleksi industri kelapa sawit 2019 dan prospek 2020). https://gapki.id/news/16190/refleksi-industri-kelapa-sawit-2019-dan-prospek-2020.

General Principles for the Addition of Essential Nutrients to Foods (CXG 9-1987). Available at http://www.fao. org/fao-who-codexalimentarius/codex-texts/guidelines/en/.

General Principles of Food Hygiene (CXC 1-1969) Available at http://www.fao. org/fao-who-codexalimentarius/codex-texts/codes-of-practice/en/.

General Standard for Contaminants and Toxins in Food and Feed (CXS 193-
1995). Available at http://www.fao. org/fao-who-codexalimentarius/codex-texts/list-standards/en/.

General Standard for Food Additives (CXS 192-1995). Available at http:// www.fao.org/fao-who-codexalimentarius/codex-texts/list-standards/en/.

Oil Market Research. 2020. Global Palm Oil Market: Insights, Trends and Forecast (2020-2024). Available at https:// www.oilandgas360.com/global-palmoil-market-insights-trends-and-forecast-2020-2024-researchandmarketscom/.

Wisena B A, Daryanto A, Arifin B and Oktaviani R. 2014. Sustainable Development Strategy and the Competitiveness of Indonesian Palm Oil Industry. IJMSR. 2:102-15. 